\title{
EDITORIAL
}

\section{Respiratory syncytial virus therapy and prophylaxis: have we finally turned the corner?}

\author{
R.G. Hegele
}

$\mathrm{t}$ is a grim reality that the text comprising introductory paragraphs to articles about respiratory syncytial virus (RSV) has changed very little over the years, the leitmotif being that RSV is a major cause of serious respiratory illnesses such as bronchiolitis and pneumonia worldwide, yet no safe, effective vaccine or good antiviral therapy is available. In this issue of the European Respiratory Journal, OLSZEWSKA et al. [1] report an exciting advance that could well be a key step toward pulling the field of anti-RSV interventions out of its doldrums. TMC353121, a remarkable "designer" molecule with a rather unremarkable name, is a potent pharmacological inhibitor of the RSV fusion $(\mathrm{F})$ protein, a viral protein crucial to initial entry of RSV into cells and in subsequent cell-to-cell spread of infection. In contrast to many other $\mathrm{F}$ inhibitors that have been developed as promising pharmaceuticals and then abandoned, OLSZEWSKA et al. [1] used a mouse model to provide compelling evidence that TMC353121 can be used either prophylactically or therapeutically to decrease RSV lung infection and virusassociated lung inflammation and histopathology. This work extends the previous success of palivizumab (Synagis $\mathbb{R}$; MedImmune, Gaithersburg, MD, USA), a humanised monoclonal antibody directed against the RSV F protein, an effective prophylactic that is used in children considered at high risk for serious RSV infections [2]. TMC353121 appears to have a suitable pharmacokinetic and safety profile and is amenable to a variety of dosing regimens. Importantly, and if TMC353121 administration is started within $48 \mathrm{~h}$ of RSV infection, the drug is an effective therapeutic agent. The 48-h time window between onset of infection and onset of treatment has bona fide practical utility for human RSV infections in the clinical setting. Administration of TMC353121 decreased RSV load by 1-2 logs at the peak of infection (day 4 post-RSV inoculation in the mouse model). Decreases in viral load were associated with a significantly attenuated host immune and inflammatory response, as assessed by lung histopathological examination, and measurement of inflammatory cells and chemical mediators in bronchoalveolar lavage fluids. RSV-induced weight loss, a characteristic of the mouse model, was also inhibited by TMC353121. As a compound effective for both prophylaxis and therapy in vivo, TMC353121 has true potential for successful translation into clinical use.

Tempering this enthusiasm is the poor track record of candidate RSV vaccines and therapeutic agents, a story characterised by a

CORRESPONDENCE: R.G. Hegele, Dept of Laboratory Medicine and Pathobiology, University of Toronto, Toronto, ON, M5S 1A8, Canada. E-mail: richard.hegele@utoronto.ca recurrent pattern of high hopes followed by disappointment. In the 1960s, a formalin-inactivated, whole virus candidate vaccine was developed using similar approaches to those used for producing spectacularly successful vaccines against measles and other viruses. In field trials, vaccine recipients developed more severe illness during natural RSV infection and several deaths occurred [3]. This tragedy put a major damper on subsequent RSV vaccine research and development, and ushered in an era of caution that persists to this day. Despite incredible efforts on the part of talented and dedicated scientists, numerous candidate RSV vaccines have failed because of an inability to achieve the optimal balance of safety and potency. A recent candidate RSV vaccine (Novavax, Inc., Rockville, MD, USA), produced by using a clever approach of making purified recombinant particles of the viral F protein, began phase 1 clinical trials in January 2011 [4]. Will this latest preparation be the definitive RSV vaccine that finally makes it to general use? Stay tuned.

An alternative strategy of using anti-RSV antibodies for prophylaxis has met with far greater clinical and commercial success. An early product approved for use in humans, respiratory syncytial virus immune globulin intravenous (RespiGam $\mathbb{}$; MedImmune), a polyclonal antibody prepared from human donors, was replaced by palivizumab (Synagis ${ }^{\circledR}$ ), a monoclonal antibody given by intramuscular injection. Despite its easier mode of administration, issues related to cost and other considerations have resulted in palivizumab being given to only an estimated 3\% of potential recipients [5]. Motavizumab (Numax ${ }_{\mathbb{B}}$; MedImmune/AstraZeneca, Macclesfield, UK), another humanised monoclonal antibody developed with the goal of being the successor to palivizumab, was withdrawn by its manufacturer, AstraZeneca, in December 2010 in response to concerns raised by the US Food and Drug Administration. The company's decision resulted in writing off $>\$ 450$ million [6], clear evidence that RSV drug development is a high-stakes endeavour with profound financial implications. In summary, use of anti-RSV antibodies for prophylaxis continues to face challenges.

What are other approaches for RSV prophylaxis or therapy, either existent or on the horizon? Ribavirin, a purine analogue, interferes with RNA metabolism required for RSV replication. Once highly touted as "gold standard" RSV therapy, clinical use of ribavirin has fallen out of favour in recent years and the drug carries potential risks, including teratogenic potential [7]. RNA interference (RNAi) is an approach that can "knock down" expression of targeted proteins. For RSV, RNAi directed against the viral nucleocapsid protein has 
been tested in a recent human clinical trial, with promising results [8].

Perhaps the emphasis on developing single agent therapy for RSV infection is misguided and requires fundamental rethinking. One reason why HIV infection is now approached as a chronic disease is because the benefits of multidrug therapy (e.g. highly active antiretroviral therapy (HAART)) were superior to monotherapy regimens [9]. For RSV, PRINCE et al. [10] pioneered the concept of polypharmacy, but in contrast to HAART, this was done from the perspective of the mechanisms of severe RSV infections involving a combination of productive viral infection and host inflammatory and immune responses. Their approach used an antiviral agent (palivizumab) in combination with an anti-inflammatory agent (corticosteroid). The concept of multiple antiviral agents used simultaneously for RSV infections has not gained much traction to date.

Drug "repurposing", defined as redeploying a drug originally developed for an unrelated clinical indication, is a proven approach in numerous patient settings but has not yet been exploited for RSV infections. For example, drug repurposing has been used successfully for treatment of malaria infections. What is particularly impressive in this instance is the short span from bench research to clinical trial validation [11]. One major advantage of drug repurposing is that safety profiles for the compounds in question have often already been established, thereby accelerating the time to clinical application. For RSV, our group has taken the approach of targeting specific aspects of host cell machinery that function as chokepoints of viral replication. Specifically, p38 mitogen-activated protein (MAP) kinase is a cell-signalling enzyme used by RSV during its replicative lifecycle [12], and we showed that treatment of cultured cells with a p38 MAP kinase inhibitor dramatically inhibited replication of several respiratory viruses (including RSV), without the drug causing overt cellular toxicity [13]. While these findings are intriguing, further studies are needed to extend our in vitro observations to the in vivo state. As an approach that focuses on the host (in addition to the virus), drug repurposing could develop into a novel antiviral strategy for RSV and other respiratory viruses. This development underscores the requirement for ongoing basic research to discover and characterise viral-host interactions as a stimulus to inform potential new uses of existing drugs.

The paucity of known cellular targets for RSV has, in a major way, slowed anti-RSV drug innovation. Surprisingly little is understood about how RSV interacts with the host cell surface to initiate infection, and the viral and host factors involved in stimulating or inhibiting viral replication. Our group has identified and carried out considerable validation of a novel cellular receptor to RSV [14] and this discovery represents a new target for development of anti-RSV interventions. Others have elucidated fascinating aspects of the host cell's production of cytoplasmic stress granules during the course of RSV infection [15] and pharmacologically modulating this cellular response for the benefit of the host is another potentially fruitful area of future research.

Overall, the essence of translational research is to take discoveries through a process of validation and qualification toward eventual clinical implementation, a necessary and challenging endeavour. OLSZEWSKA et al. [1] are to be congratulated for producing an impressive body of evidence that justifies further evaluation of TMC353121, as the compound makes its way along the difficult road toward clinical implementation. At long last, we may well have turned the corner on RSV therapy and prophylaxis.

\section{STATEMENT OF INTEREST}

None declared.

\section{REFERENCES}

1 Olszewska W, Ispas G, Schnoeller C, et al. Antiviral and lung protective activity of a novel respiratory syncytial virus fusion inhibitor in a mouse model. Eur Respir J 2011; 38: 401-408.

2 Meissner HC, Long SS. Revised indications for the use of palivizumab and respiratory syncytial virus immune globulin intravenous for the prevention of respiratory syncytial virus infections. Pediatrics 2003; 112: 1447-1452.

3 Kim HW, Canchola JG, Brandt CD, et al. Respiratory syncytial virus disease in infants despite prior administration of antigenic inactivated vaccine. Am J Epidemiol 1969; 89: 422-434.

4 Novavax moving forward with vaccine study. Associated Press. December 15, 2010. www.novavax.com/download/releases/1-411\%20RSV\%20Phase\%201\%20trial\%20FINAL2.pdf Date last updated: January 4, 2011.

5 Martin JA, Hamilton BE, Sutton PD. Births: final data for 2005. Natl Vital Stat Rep 2007; 56: 1-103.

6 Anonymous. AstraZeneca drops work on motavizumab drug. Associated Press, December 21, 2010. www.astrazeneca.com/Media/ Press-releases/Article/AstraZeneca-discontinues-motavizumab-RSV Date last updated: December 21, 2010.

7 Krilov LR. Respiratory syncytial virus disease: update on treatment and prevention. Expert Rev Anti Infect Ther 2011; 9: 27-32.

8 DeVincenzo J, Lambkin-Williams R, Wilkinson T, et al. A randomized, double-blind, placebo-controlled study of an RNAibased therapy directed against respiratory syncytial virus. Proc Natl Acad Sci USA 2010; 107: 8800-8805.

9 De Clercq E. Antiretroviral drugs. Curr Opin Pharmacol 2010; 10 507-515.

10 Prince GA, Mathews A, Curtis SJ, et al. Treatment of respiratory syncytial virus bronchiolitis and pneumonia in a cotton rat model with systemically administered monoclonal antibody (Palivizumab) and glucocorticosteroid. J Infect Dis 2000; 182: 1326-1330.

11 Boggild AK, Krudsood S, Patel SN, et al. Use of peroxisome proliferator-activated receptor gamma agonists as adjunctive treatment for Plasmodium falciparum malaria: a randomized, double-blind, placebo-controlled trial. Clin Infect Dis 2009; 49: 841-849.

12 Monick M, Staber J, Thomas K, et al. Respiratory syncytial virus infection results in activation of multiple protein kinase $\mathrm{C}$ isoforms leading to activation of mitogen-activated protein kinase. J Immunol 2001; 166: 2681-2687.

13 Marchant D, Singhera GK, Utokaparch S, et al. Toll-like receptor 4-mediated activation of p38 mitogen-activated protein kinase is a determinant of respiratory virus entry and tropism. J Virol 2010; 84: 11359-11373.

14 Tayyari F, Marchant D, Moraes TJ, et al. Identification of nucleolin as a cellular receptor for human respiratory syncytial virus. Nature Medicine 2011; (In press).

15 Lindquist ME, Lifland AW, Utley TJ, et al. Respiratory syncytial virus induces host RNA stress granules to facilitate viral replication. J Virol 2010; 84: 12274-12284. 